\title{
MODOS DE ASSISTÊNCIA VENTILATÓRIA
}

\author{
MODES OF MECHANICAL VENTILATION
}

Adriana Ignácio de Pádua1 \& José Antônio Baddini Martinez²

\begin{abstract}
${ }^{1}$ Médica Assistente. ${ }^{2}$ Docente. Divisão de Pneumologia do Departamento de Clínica Médica da FMRP - USP. CorRespondêncIA: José Antônio Baddini Martinez. Divisão de Pneumologia. Departamento de Clínica Médica. Faculdade de Medicina de Ribeirão Preto - USP. Avenida Bandeirantes, 3900. CEP 14048-900 Ribeirão Preto, São Paulo, Brasil. Telefone: (16)-6022531. Fax: 16-6336695. email: jabmarti@fmrp.usp.br
\end{abstract}

PÁDUA AI \& MARTINEZ JAB. Modos de assistência ventilatória. Medicina, Ribeirão Preto, 34: 133-142, abr./jun. 2001

RESUMO: Diversas são as modalidades de ventilação mecânica por pressão positiva atualmente disponíveis. A escolha do modo mais adequado para uma determinada situação está na dependência das características clínicas do paciente, do tipo de respirador disponível, e da experiência da equipe médica e paramédica com o seu manuseio. $O$ presente artigo fornece uma visão geral dos princípios envolvidos com a aplicação dos principais modos de assistência ventilatória, suas indicações e aplicações na prática diária, visando a que o leitor possa familiarizar-se com o manuseio dos respiradores mecânicos, elementos essenciais para a manutenção da vida em quadros de insuficiência respiratória.

UNITERMOS: Respiração Artificial. Respiradores Mecânicos. Insuficiência Respiratória.

\section{INTRODUÇÃO}

Assistência ventilatória pode ser entendida como a manutenção da oxigenação e/ou ventilação dos pacientes portadores de insuficiência respiratória aguda, de maneira artificial, até que eles estejam capacitados a reassumi-las. O moderno uso clínico dos respiradores mecânicos iniciou-se com os ventiladores à pressão negativa, em pacientes com paralisia dos músculos respiratórios, por poliomielite, através dos pulmões de aço. Muito embora a idéia de insuflar os pulmões mediante a utilização de uma pressão positiva da boca até a traquéia fosse antiga, essa prática somente começou a ser aplicada a partir da década de 1920, com o advento da anestesia geral e a entubação endotraqueal. Posteriormente, as dificuldades em se ventilar pacientes com lesões parenquimatosas, graves levaram ao desenvolvimento de aparelhos que aplicassem uma pressão positiva diretamente nas vias aéreas, os ventiladores à pressão positiva, que tiveram seu uso difundido e acabaram por ganhar uma posição de destaque no tratamento da insuficiência respiratória. Os objetivos principais do uso de suporte ventilatório, mecânico, em pacientes, encontram-se listados na Tabela I.

Temos por finalidade transmitir uma visão geral sobre ventilação mecânica e as diversas técnicas ventilatórias, empregadas para a manutenção das trocas gasosas e a preservação da microestrutura pulmonar, visando promover uma recuperação mais precoce dos quadros de insuficiência respiratória aguda.

\section{O CICLO RESPIRATÓRIO}

Didaticamente, o ciclo respiratório, durante ventilação com pressão positiva, nas vias aéreas, pode ser dividido em quatro fases (Figura 1).

I- Fase inspiratória: o respirador deverá insuflar os pulmões do paciente, vencendo as propriedades 
elásticas e resistivas do sistema respiratório. Ao final da insuflação pulmonar, uma pausa inspiratória poderá, ainda, ser introduzida, prolongando-se a fase, de acordo com o necessário para uma melhor troca gasosa.

II- Mudança da fase inspiratória para a fase expiratória: o ventilador deverá interromper a fase inspiratória (após a pausa inspiratória, quando ela estiver sendo utilizada) e permitir o início da fase expiratória; é o que se chama de ciclagem, dispondo-se hoje de ciclagem por critérios de pressão, fluxo, volume e tempo.

III - Fase expiratória: o ventilador deverá permitir o esvaziamento dos pulmões, normalmente, de forma passiva.

IV - Mudança da fase expiratória para a fase inspiratória: essa transição pode ser desencadeada pelo ventilador ou pelo paciente. É o que se chama de ciclo respiratório, dispondo-se, hoje, de mecanismos de disparo por tempo, pressão ou fluxo.

Na observação do ciclo respiratório mecânico, uma série de parâmetros ventilatórios podem ser identificados.

Volume Corrente $\left(V_{T}\right)$ : corresponde ao volume de gás movimentado durante uma respiração. Em con-

\section{Tabela I: Objetivos da Ventilação Mecânica}

- Melhorar as trocas gasosas

Reverter a hipoxemia.

Atenuar a acidose respiratória aguda.

- Atenuar a dificuldade respiratória Diminuir o consumo de oxigênio relacionado à respiração.

Reverter a fadiga muscular respiratória.

- Alterar as relações pressão-volume Evitar ou reverter atelectasias. Melhorar a complacência pulmonar. Evitar a progressão da lesão pulmonar.

- Permitir a reparação dos pulmões e vias aéreas

- Evitar complicações

dições fisiológicas de repouso, para um adulto normal, gira em torno de $500 \mathrm{ml}$. Muito embora, até um passado recente, fossem empregados, em ventilação mecânica, volumes correntes elevados (10-15 $\left.\mathrm{ml} . \mathrm{Kg}^{-1}\right)$, a abordagem atual é manter o volume em valores menores, em torno de $6-10 \mathrm{ml} \cdot \mathrm{Kg}^{-1}$.

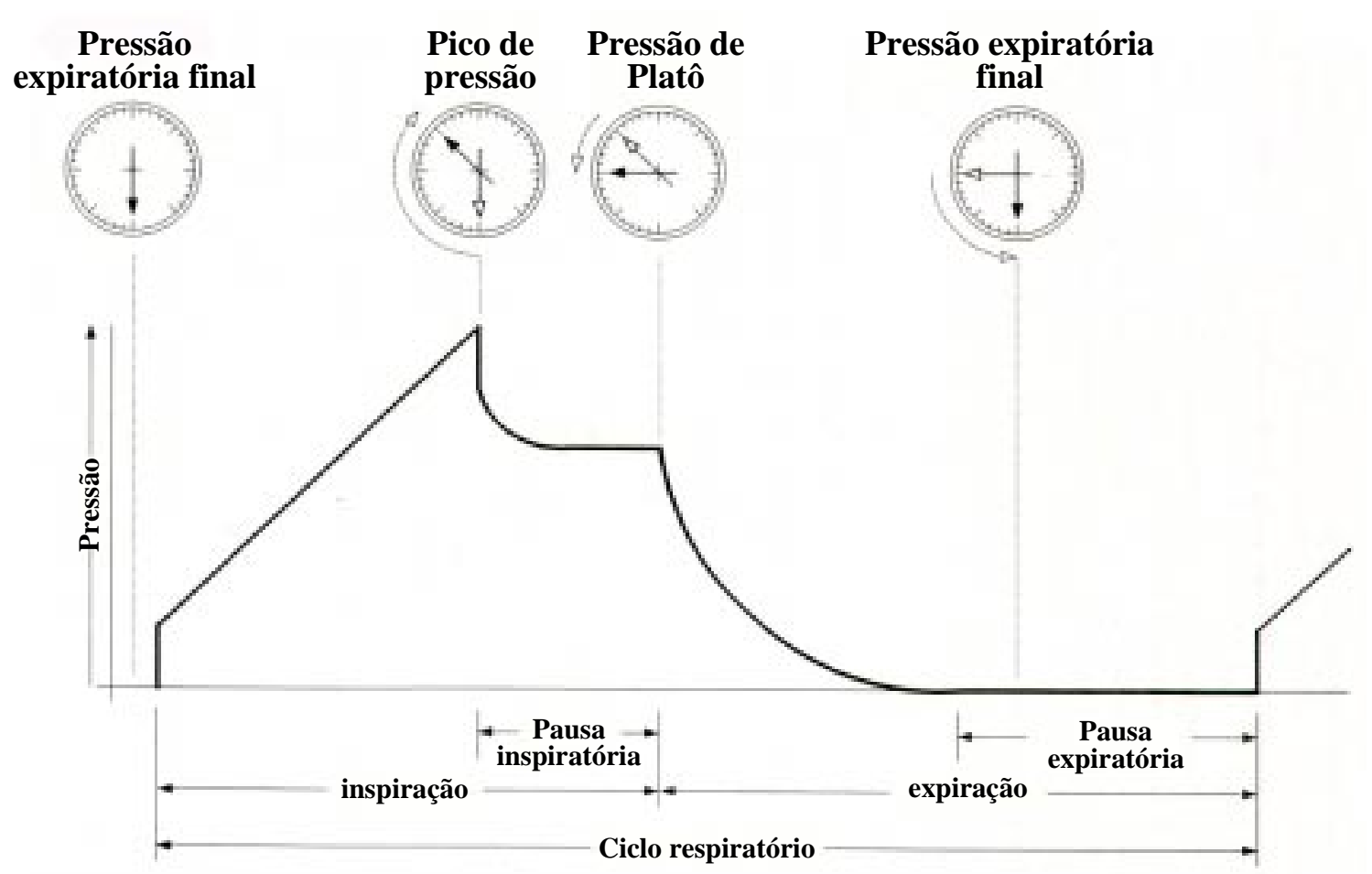

Figura 1: Ciclo respiratório. Modificado de referência 4. 
Freqüência Respiratória (f): número de incursões respiratórias que o paciente apresenta por minuto. Valores fisiológicos giram em torno de 10 a 20 incursões por minuto (ipm).

Volume Minuto $\left(V_{E}\right)$ : volume total de gás mobilizado durante um minuto. É calculado pela fórmula $\mathrm{V}_{\mathrm{E}}=\mathrm{fx} \mathrm{V}_{\mathrm{T}}$ e seus valores fisiológicos giram em torno de $7,5 \mathrm{~L} / \mathrm{min}$.

Tempo Inspiratório $\left(T_{I}\right)$ : tempo que leva para a inspiração se completar. Geralmente, gira em torno de um terço do ciclo respiratório.

Tempo Expiratório $\left(T_{E}\right)$ : tempo gasto para a expiração se completar. Geralmente, gira em torno de dois terços do ciclo respiratório.

Tempo Total $\left(T_{T o t}\right)$ : tempo de duração de um ciclo respiratório completo. $\mathrm{T}_{\mathrm{Tot}}=\mathrm{T}_{\mathrm{I}}+\mathrm{T}_{\mathrm{E}}$.

Fluxo inspiratório $\left(V_{I}\right)$ : volume de gás que passa pela via de saída inspiratória do ventilador, na unidade de tempo. Corresponde à velocidade com que o gás entra no paciente, expressa em litros por minuto.

Pico de Pressão Inspiratória (PIP): é o maior valor de pressão atingido durante a inspiração do $\mathrm{V}_{\mathrm{T}}$, durante um ciclo de ventilação mecânica. Valores excessivos, geralmente além de $50 \mathrm{~cm} \mathrm{H}_{2} \mathrm{O}$, podem cursar com traumas associados à ventilação mecânica, tais como pneumotórax e pneumomediastino.

Pausa Inspiratória: período curto de tempo, correspondente à oclusão da via de saída expiratória, do respirador, impedindo temporariamente o início da expiração. É um mecanismo empregado para prolongar o $\mathrm{T}_{\mathrm{I}}$.

Pressão de "Plateau": valor da pressão das vias aéreas, medida no momento da pausa inspiratória. Admite-se que seja o parâmetro que melhor reflita as pressões alveolares no momento do término da insuflação pulmonar. Valores muito altos de pressão de plateau, geralmente, além de $35 \mathrm{~cm} \mathrm{H}_{2} \mathrm{O}$, associem-se a lesão parenquimatosa, pulmonar, induzida pela ventilação mecânica.

Pressão Expiratória $(P E)$ : corresponde à pressão observada nas vias aéreas, ao final da expiração. Habitualmente, ela cai a zero, denominada, então, pressão expiratória (ZEEP), mas podem ser feitos ajustes nos ventiladores para que ela atinja valores positivos (PEEP).

Pressão Expiratória Positiva Final (PEEP): aplicação, nas vias aéreas, de uma pressão positiva, constante, ao final da expiração. Sua aplicação tem por finalidade reduzir os distúrbios das trocas gasosas, permitindo aos pacientes a administração de uma menor fração inspirada de oxigênio. Admite-se que seus efeitos terapêuticos se devam à abertura de pequenas vias aéreas e espaços alveolares colabados, ou, ainda, às suas repercussões hemodinâmicas. Alguns autores advogam que, mesmo quando ventilando pulmões normais, deva-se aplicar um nível baixo de PEEP, o chamado "PEEP fisiológico" (3-5 $\mathrm{cm} \mathrm{H}_{2} \mathrm{O}$ ). Quando ventilando pulmões com baixa complacência, valores elevados de PEEP podem ser necessários (5-20 cm $\left.\mathrm{H}_{2} \mathrm{O}\right)$. Nessa última situação, é mais adequado orientar-se pela confecção de curvas pressãovolume com a detecção dos chamados pontos de inflexão, mínimo e máximo.

Fração Inspirada de Oxigênio $\left(\mathrm{F}_{I} \mathrm{O}_{2}\right)$ : conteúdo de oxigênio na mistura gasosa, administrada ao paciente. Pode variar entre 0,21 e 1,0. Vale a pena lembrar que o uso de oxigênio em frações inspiradas, elevadas, além de 0,6 , por longos períodos de tempo, pode levar a lesão tóxica pulmonar.

\section{MECANISMOS DE CICLAGEM DOS APA- RELHOS}

As maneiras pelas quais os ventiladores são projetados para interromper a fase inspiratória e dar início à fase expiratória, recebem o nome de modos de ciclagem do respirador. A transição pode ocorrer basicamente através de quatro mecanismos:

Ciclagem a tempo: a transição inspiração/expiração ocorre após um período de tempo pré-fixado e ajustável no ventilador. É o padrão comumente encontrado nos ventiladores infantis (geradores de pressão não constante) e na ventilação com pressão controlada (gerador de pressão constante). Nessas duas situações, o volume corrente não pode ser diretamente controlado, sendo uma conseqüência do tempo inspiratório, programado, assim como da pressão aplicada e da impedância do sistema respiratório.

Ciclagem a volume: o final da fase inspiratória ocorre, quando é atingido um volume pré-ajustado de gás, comumente sinalizado por um fluxômetro, localizado no circuito inspiratório do aparelho. Esse tipo de ventilação não permite um controle direto sobre as pressões geradas em vias aéreas, o que faz com que muitos desses ventiladores incorporem uma válvula de segurança nos sistemas de alarme de pressão, capaz de abortar a fase inspiratória sempre que a pressão ultrapassar determinados níveis.

Ciclagem a pressão: o final da fase inspiratória é determinado pelo valor de pressão alcançado nas 
vias aéreas. Quando a pressão atinge o valor prefixado e ajustável interrompe-se a inspiração, independentemente do tempo inspiratório gasto para atingir aquela pressão. Tais ventiladores são susceptíveis às variações de complacência e resistência do sistema respiratório, podendo ocorrer uma drástica redução de volume corrente na vigência, por exemplo, de um broncoespasmo.

Ciclagem a fluxo: o fim da fase inspiratória ocorre a partir do momento em que o fluxo inspiratório cai abaixo de níveis críticos, independentemente do tempo transcorrido ou do volume liberado para o paciente. A grande característica desse dispositivo é a de permitir ao paciente exercer um controle efetivo sobre o tempo e o pico de fluxo inspiratório, e, ainda, sobre o seu volume corrente. A escolha do nível crítico de fluxo que desativa a fase inspiratória varia de ventilador para ventilador, existindo, porém, uma certa padronização (normalmente $25 \%$ do pico de fluxo, ou um valor fixo entre 6 e 10 litros/minuto, por exemplo).

\section{MODOS DE VENTILAÇÃO MECÃNICA}

\subsection{Modos Convencionais}

Ventilação mecânica volume controlada $(C M V)$ : nesse modo de ventilação, a freqüência respiratória e o volume corrente são constantes e prédeterminados. O ventilador inicia a inspiração seguinte após um tempo estipulado, estabelecido a partir do ajuste do comando da frequiência respiratória (Figura 2). Na maioria dos ventiladores, quando se ativa o comando de ventilação controlada, todos os demais mecanismos de disparo e o comando de sensibilidade ficam desativados. Esse modo ventilatório está indicado para pacientes com mínimo ou nenhum esforço respiratório, por disfunção do sistema nervoso central como, por exemplo, na síndrome de Guillain-Barré ou em casos de intoxicação exógena por drogas. Também é utilizado quando a respiração está suprimida intencionalmente devido a anestesia, sedação ou bloqueio neuromuscular. Ainda se mostra útil em situações nas quais o esforço inspiratório negativo é contra-indicado, como em alguns casos de traumas torácicos graves. A ventilação controlada também pode ser administrada numa forma limitada, por pressão. Nessa modalidade, denominada pressão controlada, os parâmetros respiratórios são igualmente constantes e previamente estabelecidos pelo aparelho. Dá-se preferência a essa modalidade, quando desejamos limitar as pressões inspiratórias máximas no circuito e o risco do surgimento de barotrauma, bem como na vigência de pulmões pouco complacentes. Entretanto, como o parâmetro primário, determinante do final da inspiração, é uma pressão pré-estabelecida, o volume corrente pode sofrer indesejáveis variações, em função da presença de secreções respiratórias e alterações da complacência torácica.

Ventilação assistida/controlada $(A / C)$ : nessa modalidade, o ventilador permite um mecanismo misto de disparo da fase inspiratória por tempo ou pressão. Enquanto o disparo por pressão é ativado pelo esforço inspiratório do paciente (assistido), o disparo por tempoé deflagrado pelo aparelho (controlado), funcionando como um mecanismo de resgate, que é ativado apenas quando o ciclo assistido não ocorre, garantindo uma freqüência mínima (Figura 3). Sempre que se utiliza a modalidade $\mathrm{A} / \mathrm{C}$, o comando do ventilador chamado sensibilidade é acionado, devendo-se optar por um valor dentro de uma escala fornecida pelo aparelho em questão. O ajuste da sensibilidade consiste no controle do nível de esforço inspiratório, necessário para acionar a fase inspiratória. A ventilação $\mathrm{A} / \mathrm{C}$ está indicada em situações nas quais o estímulo neural res-

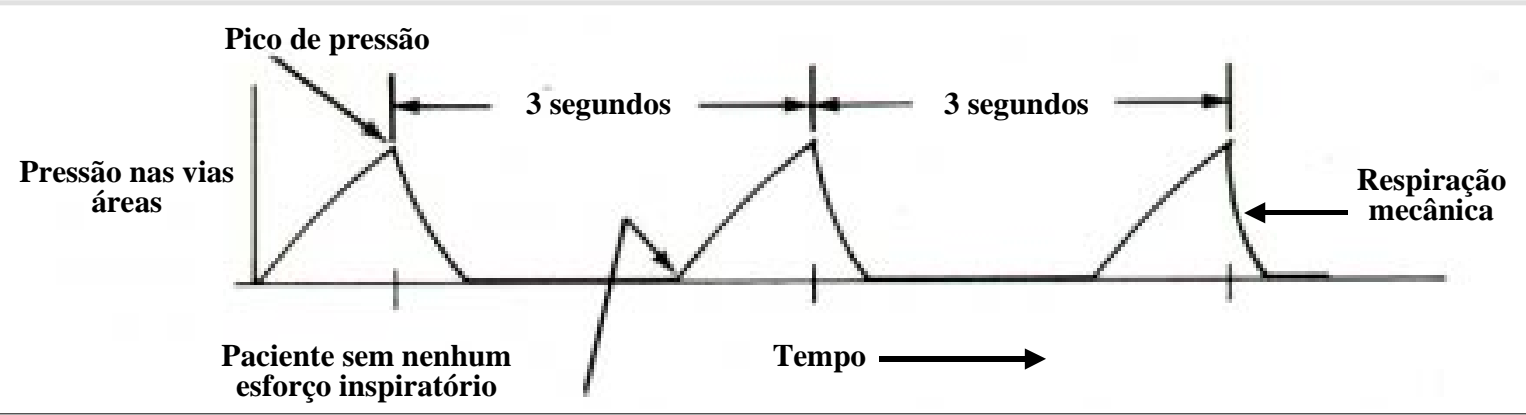

Figura 2: Ventilação Mecânica Controlada (VMC). A freqüência respiratória é estipulada dentro de um intervalo de tempo. Não ocorre esforço respiratório, espontâneo do paciente. Modificado de referência 4. 


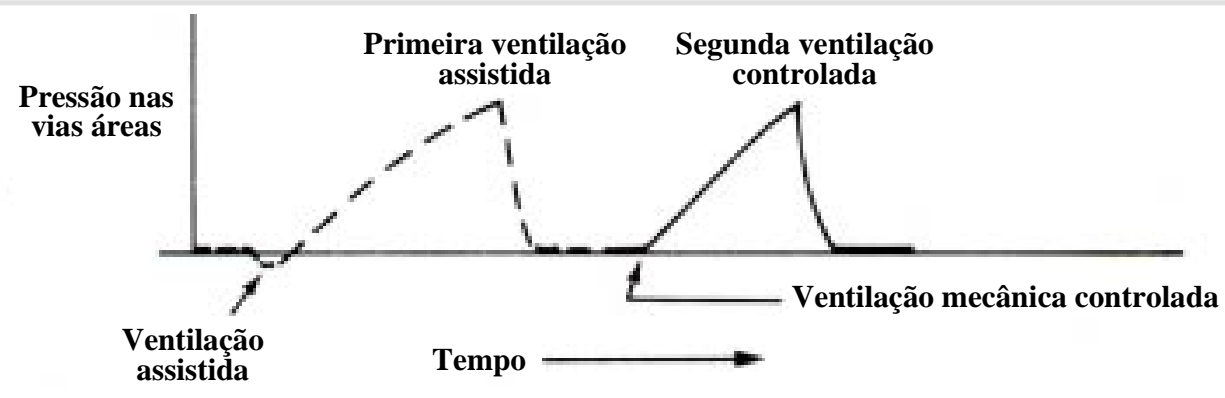

Figura 3: Ventilação Mecânica, Assistida, Controlada (VMAC). O paciente recebe um número estipulado de incursões respiratórias e pode desencadear ciclos mecânicos, assistidos, adicionais. Observe a deflexão negativa, quando o paciente realiza um esforço respiratório. Modificado de referência 4.

por exemplo, nos quadros de insuficiência respiratória com complacência pulmonar muito diminuída.

Ventilação mandatória intermitente (IMV): alguns ventiladores permitem a combinação dos modos assistido/controlado com períodos de ventilação espontânea. No IMV, o paciente recebe um número fixo e pré-determinado de um $\mathrm{V}_{\mathrm{T}}$ estabelecido.

piratório (drive) é normal, embora os músculos respiratórios não estejam totalmente aptos para o trabalho como, por exemplo, em pacientes recuperando-se de anestesias. Essa modalidade também costuma ser empregada quando o drive respiratório é normal mas os músculos respiratórios estão insuficientes para empreender todo o trabalho respiratório necessário como,
Nos intervalos das respirações mandatórias, o paciente pode iniciar respirações espontâneas, cujos volumes estão na dependência do grau de esforço respiratório do indivíduo (Figura 4a). Os ciclos espontâneos podem ser auxiliados por alguns dispositivos que permitam uma ventilação muito similar à ventilação em ar ambiente, ou mesmo serem auxiliados por um

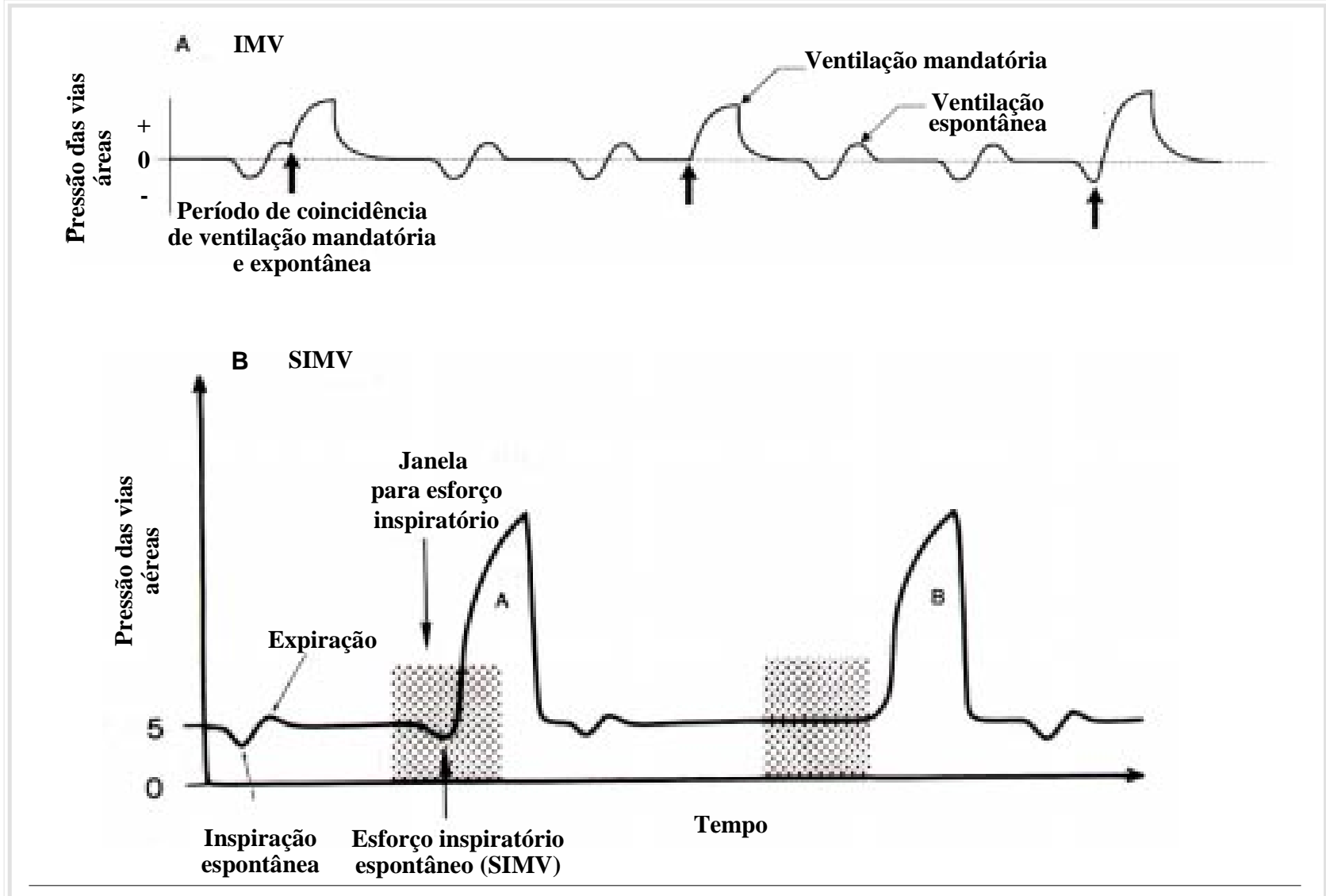

Figura 4: A) IMV - a respiração mandatória pode coincidir com períodos de ventilação espontânea. B) SIMV - a ventilação mandatória ocorre em sincronia com a respiração espontânea do paciente. Modificado de referência 4. 
certo nível de pressão contínua de vias aéreas (CPAP) ou pressão de suporte. A chamada ventilação mandatória intermitente sincronizada (SIMV) difere do IMV pelo fato de, ao invés do $\mathrm{V}_{\mathrm{T}}$ mandatório ser administrado a um tempo preciso, independentemente da fase do ciclo respiratório do paciente, o ventilador fornecer tal volume no momento de esforço respiratório do doente. Para tanto, o ventilador monitora os esforços respiratórios dos indivíduos periodicamente, dentro de uma janela de tempo. Dentro dessa janela, no momento do esforço inspiratório do paciente, é desencadeada uma inspiração. Caso o paciente não inspire, será fornecida uma ventilação mandatória, dentro do tempo pré-estabelecido (Figura 4b). O SIMV é um mecanismo mais vantajoso, pois evita que o aparelho inicie um fluxo inspiratório num momento em que o paciente poderia estar expirando num ciclo espontâneo. Tanto o IMV como o SIMV podem ser usados em pacientes com drive respiratório normal, porém com músculos ainda não completamente aptos para a demanda de trabalho. Muito embora tenham sido descritos inicialmente como estratégias auxiliares no processo de desmame, eles podem ser utilizados como métodos ventilatórios isolados, em pacientes com drive respiratório normal e complacência pulmonar pouco alterada.

Pressão positiva contínua nas vias aéreas (CPAP): nesse tipo de ventilação, o doente respira espontaneamente através do circuito pressurizado do aparelho, de tal forma que uma certa pressão positiva, definida quando do ajuste do respirador, é mantida praticamente constante durante todo o ciclo respiratório (Figura 5). Para ser utilizado, esse método necessita de doentes com capacidade ventilatória mantida, geralmente sendo empregado em pacientes com patologias parenquimatoreferência 4 . sas, puras, de pouca gravidade e/ou no processo de desmame. É uma técnica utilizada com a finalidade de aumentar a capacidade residual, funcional, pulmonar e melhorar a oxigenação arterial, com poucos efeitos sobre as trocas do $\mathrm{CO}_{2}$. A aplicação de CPAP pode ser feita inclusive em pacientes extubados, através de máscaras acopladas a dispositivos mecânicos especiais.

Pressão de suporte (PSV): é um modo recente de ventilação mecânica, e consiste na aplicação de níveis pré-determinados de pressão positiva e constante nas vias aéreas do doente, apenas durante a fase inspiratória (Figura 6). O objetivo do fornecimento dessa pressão seria reduzir o trabalho dos músculos

Figura 5: Pressão positiva continua nas vias aéreas (CPAP) - o paciente respira espontaneamente

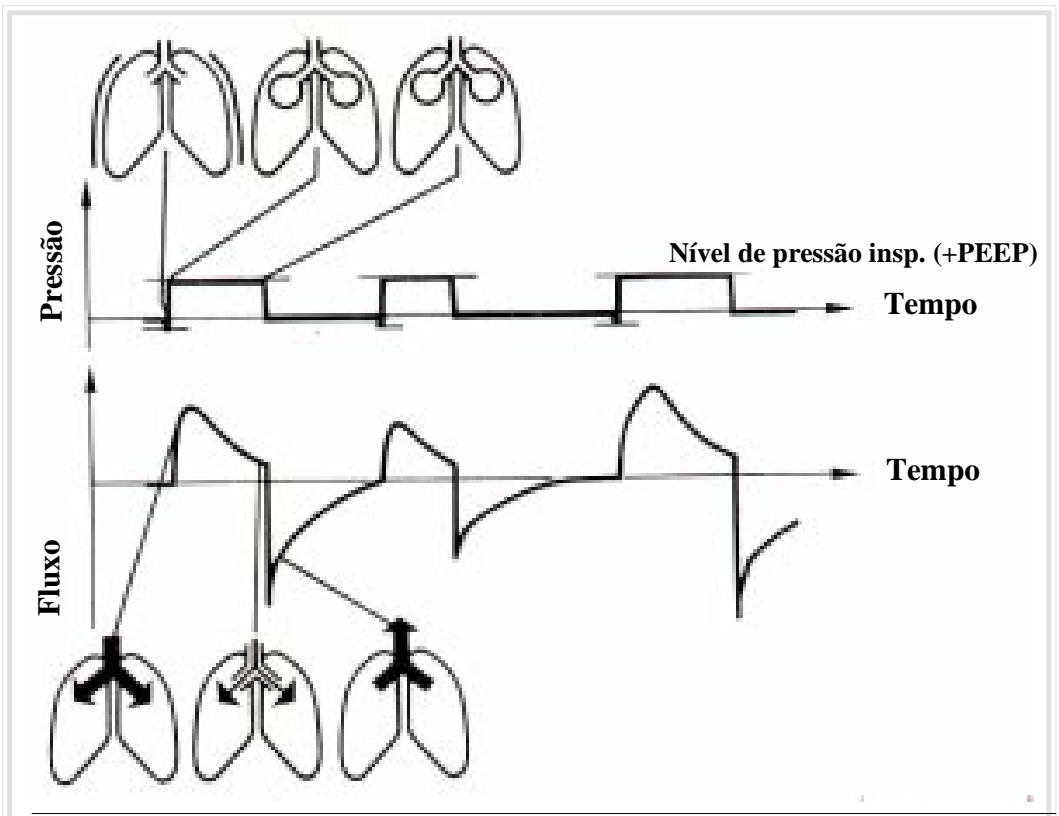

Figura 6: Ventilação com Pressão de Suporte (PSV). O paciente recebe um incremento de pressão durante a inspiração pelo fornecimento de um alto fluxo inspiratório de gás. Modificado de referência 4. 
inspiratórios (preservando a musculatura respiratória), mas, ficando ainda a cargo do doente o controle do tempo, fluxo e volume inspiratórios, assim como da própria freqüência respiratória. É um modo obrigatoriamente assistido, onde o ventilador necessita reconhecer o início de uma inspiração espontânea para ativar a pressão de suporte. Para que o respirador note o momento em que o doente finaliza sua inspiração espontânea, convencionou-se programar os aparelhos para interromper a pressão de suporte assim que o fluxo inspiratório caia abaixo de determinados níveis críticos, geralmente $25 \%$ do valor máximo daquela incursão. Altos níveis de pressão de suporte (15-20 $\left.\mathrm{cm} \mathrm{H}_{2} \mathrm{O}\right)$, associados ou não a um certo nível de PEEP, podem ser muito úteis na insuficiência respiratória, aguda, parenquimatosa, permitindo melhor sincronia com o aparelho, com diminuição do trabalho muscular respiratório, menor pico de pressão inspiratória para um mesmo volume corrente, e diminuição do tempo inspiratório devido aos altos fluxos inspiratórios alcançados. Como desvantagens, nessas situações, temos a possibilidade de deterioração das trocas gasosas, e a impossibilidade de assegurar-se um valor mínimo de volume corrente ou freqüência respiratória, em indivíduos instáveis. A PSV freqüentemente é usada ao longo do processo de desmame de ventiladores, geralmente em níveis inferiores a 15 $\mathrm{cm} \mathrm{H}_{2} \mathrm{O}$. Quando bem utilizada, pode abreviar o tempo de desmame ou mesmo aumentar suas chances de êxito, quando comparada ao SIMV e ao tubo $\mathrm{T}$, pois permite uma transição gradual da ventilação assistida para a espontânea.

Ventilação com pressão de suporte e volume garantido (VAPSV): a utilização da PSV, em doentes graves e instáveis, pode ser problemática. A ven- tilação alveolar, nessa modalidade, é conseqüência de diversas variáveis, entre elas do esforço muscular do doente e da impedância do sistema respiratório. Situações de instabilidade do drive ventilatório ou de alterações súbitas na impedância do sistema respiratório, ou mesmo situações em que um rígido controle da $\mathrm{PaCO}_{2}$ é necessário (como em casos com hipertensão intracraniana associada) são condições de uso limitado da PSV. Numa tentativa de se evitar tais deficiências, desenvolveu-se uma técnica de ventilação, que combina a pressão de suporte e a ventilação ciclada a volume num mesmo ciclo respiratório, chamada VAPSV. Funcionando através de um sistema de circuitos paralelos, ao mesmo tempo em que o paciente recebe uma pressão de suporte com fluxo livre por umas das vias do circuito, oferece-se um fluxo quadrado e fixo pela outra via (Figura 7). Portanto, com essa abordagem, pode-se estender as vantagens da PSV a situações clínicas instáveis, quando a ventilação ciclada por volume proporciona a segurança de uma ventilação alveolar mínima.

\subsection{Métodos não convencionais}

Pressão controlada com relação inspiratória/expiratória (I/E) invertida: visa ao aumento da

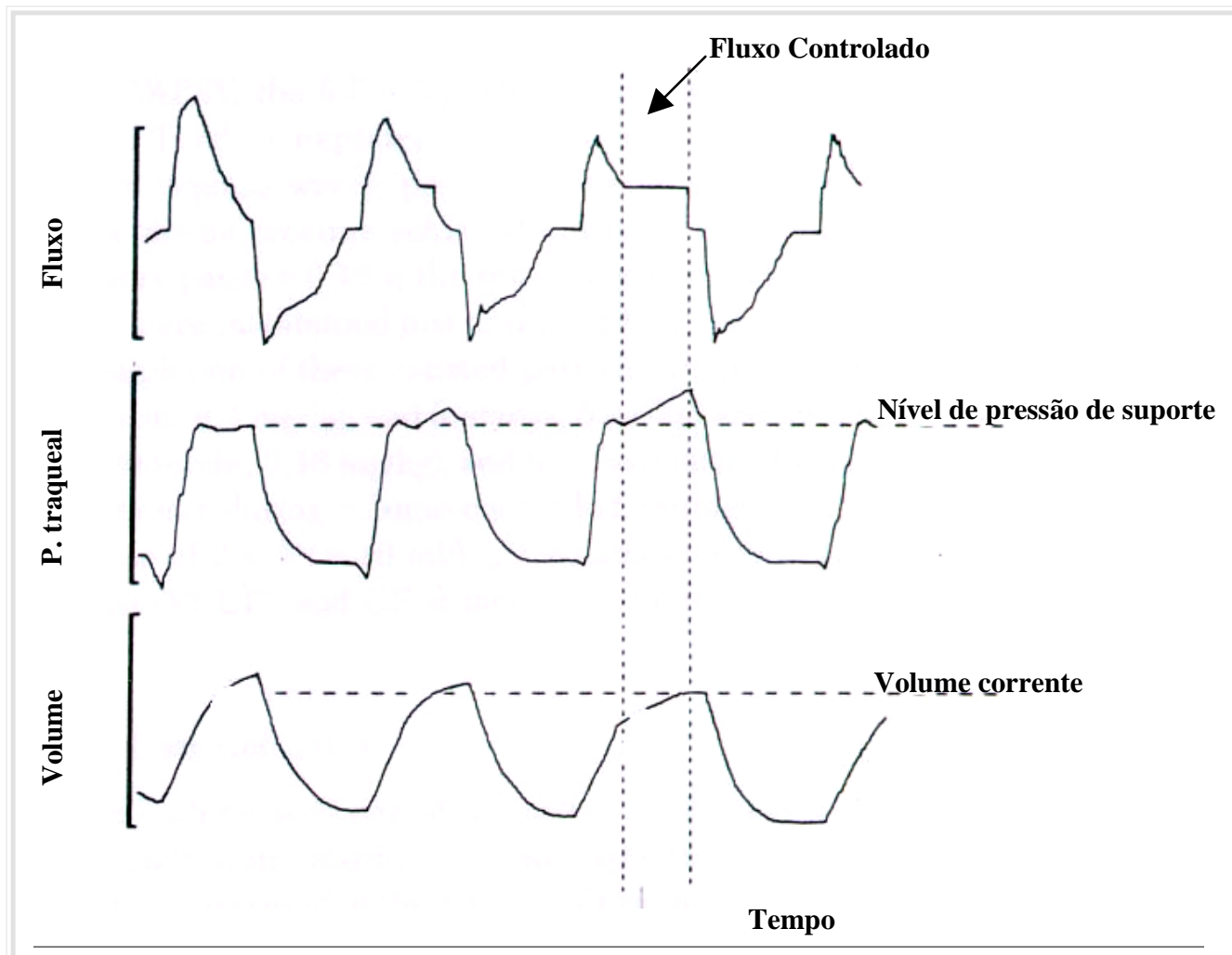

Figura 7: Ventilação de Suporte com Volume Assistido (VAPS). Modificado de referência 4. 
eficácia das trocas gasosas pelo prolongamento do tempo inspiratório. Em condições fisiológicas, a expiração possui uma duração maior do que a inspiração. Geralmente, em se tratando de ventilação mecânica, procuramos manter tal relação em torno de 1:2 e 1:3. Quando aplicamos a estratégia da relação invertida, tais razões tornam-se algo em torno de $2: 1$ ou 3:1. Essa tática leva ao surgimento do chamado "auto-PEEP", que pode ser definido como a ocorrência de pressão expiratória final, em vias aéreas distais, mais positiva do que em vias aéreas proximais (Figura 8). Em outras palavras, ao final da expiração, a pressão final das vias aéreas não chega a zero. Como conseqüência, há aumento da capacidade residual, funcional, pulmonar e abertura de pequenas vias aéreas colabadas. Essa abordagem está indicada em casos graves de insuficiência respiratória, aguda, parenquimatosa, associados a valores muito baixos de complacência e dificuldades de oxigenação. A sua introdução, geralmente, permite ciclar com um pico de pressão inspiratória menor, se comparado com abordagens tradicionais, e uma redução da $\mathrm{F}_{\mathrm{I}} \mathrm{O}_{2}$. $\mathrm{O}$ método deve ser iniciado com o paciente sedado e curarizado, com a $\mathrm{F}_{\mathrm{I}} \mathrm{O}_{2}$ igual a 1, ajustando-se o nível de I/E inicialmente em $2 / 1$. Seu uso rotineiro é dificultado por não ser um modo disponível em todos os tipos de respiradores, pela necessidade de sedação profunda, curarização e monitorização rigorosa. Além disso, como é uma modalidade pressão controlada, não garante a administração de um volume corrente constante.

Ventilação pulmonar independente: modalidade ventilatória, em que a utilização de uma sonda brônquica (também denominada de dupla luz) especial permite ventilação pulmonar em separado, pelo emprego de dois respiradores (Figura 9). Ajustes de parâmetros respiratórios tais como volume corrente e PEEP podem ser individualizados para as particularidades fisiopatológicas de cada pulmão. Está indicada em casos nos quais a lesão pulmonar concentra-se em um hemitórax como, por exemplo, contusão pulde referência 4. monar unilateral, pneumonia aspirativa, pneumonia lobar unilateral, atelectasia refratária, fistulas, etc. Um número expresivo de dificuldades associa-se com sua instalação, entre elas a necessidade de monitoração mais rigorosa devido ao uso de dois respiradores. Cuidados especiais devem ser tomados, visando à manutenção da sonda brônquica, constantemente em posição adequada. Além disso, as sondas brônquicas uti-

Figura 8: Ventilação com pressão controlada e relação inspiratória e expiratória invertida. Modificado

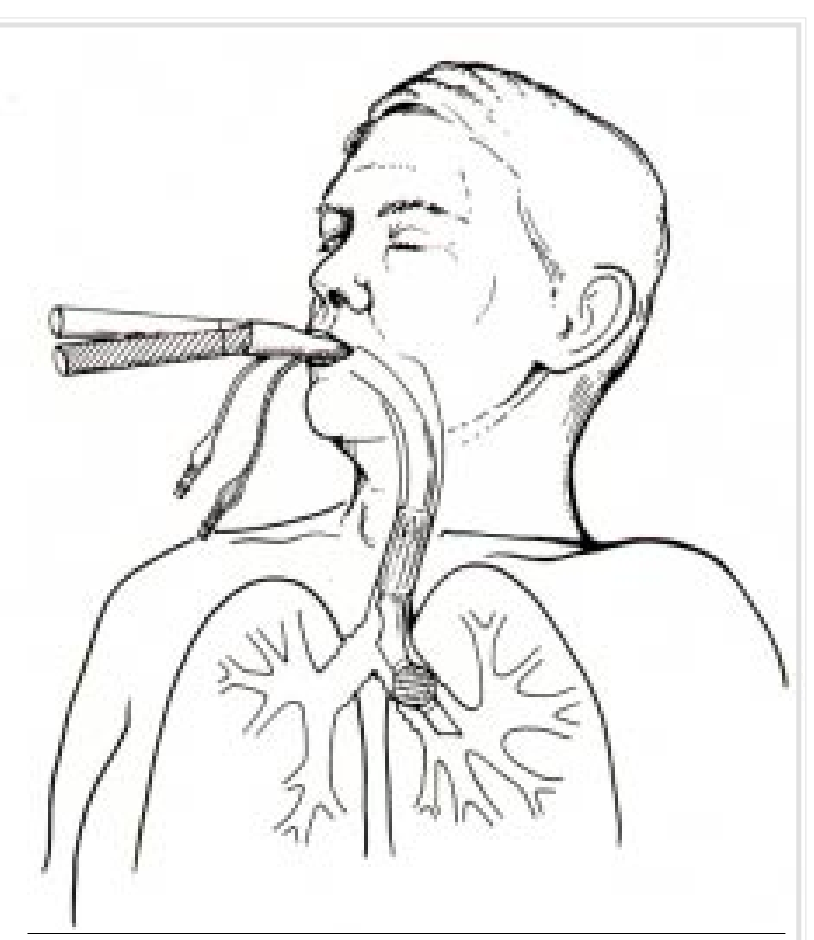

Figura 9: Ventilação Pulmonar Independente - emprega-se uma sonda brônquica ou de dupla luz, especial, permitindo a ventilação de cada pulmão isoladamente, utilizando-se dois respiradores simultaneamente. Modificado de referência 4. 
lizadas, geralmente, tem diâmetro externo elevado e diâmetro interno reduzido, o que costuma elevar a dificuldades para aspiração de secreções e risco do surgimento de rolhas de catarro.

Insuflação de gás intratraqueal (TGI): consiste na injeção de um gás através de um cateter diretamente nas vias aéreas, habitualmente oxigênio, em associação a uma forma tradicional de ventilação mecânica como, por exemplo, modo assistido/controlado. O cateter deve ser colocado em posição pré-carinal e o gás pode ser injetado durante a inspiração e a expiração. Dessa maneira, promove-se uma redução do espaço morto tanto pela diluição do $\mathrm{CO}_{2}$, que permaneceria no espaço morto, anatômico, durante a expiração, como, também, pela geração de fluxo turbulento, no local de saída do gás pelo cateter. Essa abordagem está indicada em casos que cursem com grande aumento do espaço morto e elevações indesejadas dos níveis de $\mathrm{PaCO}_{2}$. Algumas complicações, potencialmente associadas com seu uso são o surgimento de auto-PEEP, lesões da mucosa no local de contato com a extremidade do cateter, surgimento de rolhas de muco, se o gás administrado for pouco umidificado, e elevações do volume corrente, expirado.

Hipercapnia permissiva: é uma estratégia ventilatória, desenvolvida visando reduzir os riscos do surgimento de trauma pulmonar, associado a altas pressões inspiratórias, em vias aéreas. As pressões médias das vias aéreas são reduzidas pela utilização de pequenos volumes correntes, geralmente próximos a $6 \mathrm{ml} \cdot \mathrm{Kg}^{-1}$. A redução das pressões inspiratórias, em um pulmão com lesão parenquimatosa e baixa complacência, levará a uma diminuição da hiperdistensão, em áreas de alvéolos preservados. Como resultado, admitese que haverá uma redução das condições propícias ao desenvolvimento de lesão pulmonar, aguda, induzida pela ventilação. A principal conseqüência do seu emprego são elevações da $\mathrm{PaCO}_{2}$ a níveis tão elevados como 50-100 $\mathrm{mmHg}$. Essa estratégia pode ser aplicada com qualquer respirador, mais freqüentemente sendo associada ao modo de pressão controlada com PEEP. Está indicada em casos de insuficiência respiratória, aguda, parenquimatosa, grave, com baixa complacência, que estejam requerendo pressões de plateau acima de $30-35 \mathrm{~cm} \mathrm{H}_{2} \mathrm{O}$ para ventilação adequada. É uma abordagem contra-indicada em pacientes com hipertensão intra-craniana, grave instabilidade hemodinâmica e acidose metabólica prévia.

\section{VENTILAÇÃO MECÂNICA NÃO INVASIVA}

A ventilação não invasiva é definida como uma técnica em que não é empregado qualquer tipo de prótese traqueal (tubo orotraqueal, nasotraqueal ou cânula de traqueostomia), sendo a conexão entre o respirador e o paciente feita através do uso de uma máscara. É uma abordagem respiratória que vem ganhando grande popularidade nos últimos anos, podendo ser utilizada em situações tão diversas quanto a insuficiência isolada dos músculos respiratórios, apnéia do sono, ou como método de desmame. Existem aparelhos que fornecem o mesmo nível de pressão tanto na inspiração como na expiração e, portanto, são conhecidos como aparelhos de CPAP. Normalmente, eles são utilizados em casos que cursam com lesão parenquimatosa, pulmonar, de grau leve a moderado, como

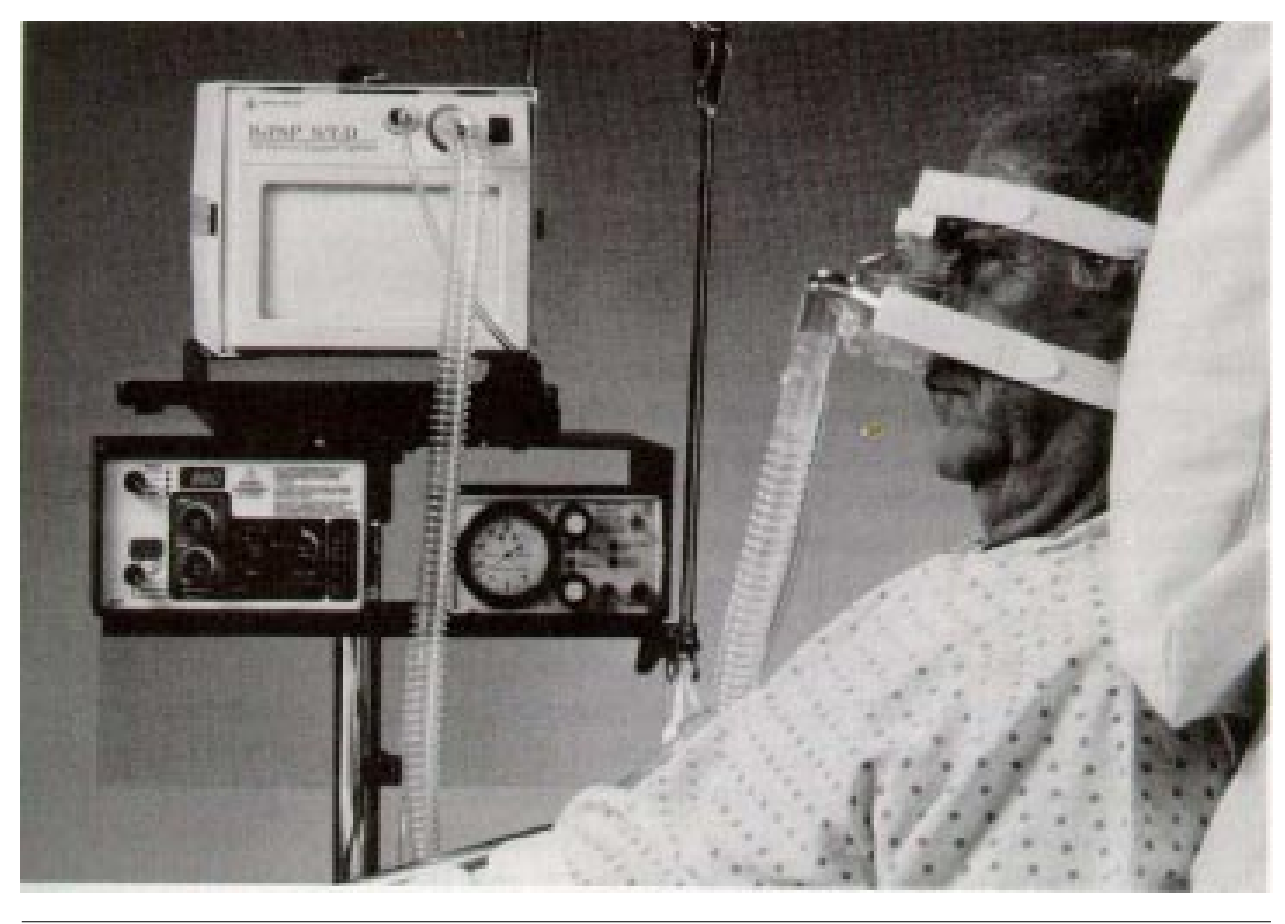

Figura 10: Ventilação mecânica não invasiva. BIPAP com máscara nasal. Modificado de referência 4. 
um expediente, visando evitar a intubação. Uma outra indicação bastante comum de CPAP é durante o repouso de pacientes com apnéia obstrutiva do sono.

Dispositivos mais modernos são capazes de fornecer ao paciente valores diferenciados de pressão, durante a inspiração (IPAP) e a expiração (EPAP). Esta última condição, normalmente, é chamada de ventilação com pressão positiva, nas vias aéreas em dois níveis (BIPAP). Em comparação com a ventilação convencional, a IPAP seria equivalente à pressão de suporte e a EPAP seria similar ao PEEP (Figura 10). O volume corrente, gerado nessas condições, fica, portanto, na dependência do fluxo respiratório, produzido pelo paciente, sua frequiência respiratória e o gradiente de pressão ajustado entre o IPAP e o EPAP. O BIPAP tem-se mostrado particularmente útil no manuseio de pacientes com doença pulmonar, obstrutiva, crônica, em fase de agudização, asma grave, quadros neuromusculares, ventilação noturna, em alguns casos de apnéia obstrutiva do sono, e como método de transição após extubação antes do paciente iniciar ventilação espontânea.

A ventilação não invasiva está contra-indicada na presença de parada respiratória, instabilidade hemodinâmica grave, pacientes não cooperativos, alto risco de vômitos e aspiração, história recente de cirurgia facial, gástrica ou esofágica, e lesões traumáticas de face. Outras condições que dificultam seu uso são pacientes ansiosos, presença de secreções abundantes e obesidade mórbida.

\section{CONCLUSÃO}

Diversas são as modalidades de ventilação mecânica por pressão positiva, atualmente disponíveis. A escolha do modo mais adequado para uma determinada situação está na dependência das características clínicas do paciente, do tipo de respirador disponível, e da experiência da equipe médica e paramédica com o seu manuseio.

A ventilação de alta freqüência, o uso de oxigenador de membrana e a remoção de $\mathrm{CO}_{2}$ através de circulação extracorpórea, embora não incluídos nesta revisão, são recursos terapêuticos que podem vir a ser utilizados em casos selecionados de insuficiência respiratória, aguda. A ventilação líqüida com perfluorcarbono é uma modalidade terapêutica, ainda em fase inicial de emprego em humanos, que guarda o potencial de revolucionar as tradicionais abordagens ventilatórias.

PÁDUA AI \& MARTINEZ JAB. Modes of mechanical ventilation. Medicina, Ribeirão Preto, 34: 133-142, apr./ june 2001.

ABSTRACT: There are several mechanical ventilation modalities available nowadays. The choice of the most apropriate ventilatory approach in a specific clinical situation depends on the features of the patient, type of ventilator available, and the experience of the medical and paramedical teams in handling the machine. This article gives a general view about the most important ventilatory modalities, the principles involved in their design, indications, and use in the daily clinical setting, aiming to help the reader to become familiarized with handling mechanical ventilators, essential tools to keep alive patients with respiratory failure.

UNITERMS: Respiration, Artificial. Ventilators, Mechanical. Respiratory Insufficiency.

\section{BIBLIOGRAFIA RECOMENDADA}

1 - AMATO MBP; VARGAS CSV; BONASSA J; SALDIVA PHN; ZIN WA \& CARVALHO CRR. Volume-assured pressure support ventilation (VAPSV). A new approach for reducing mauscle workload during acute respiratory failure. Chest 102: 1225-1234, 1992.

2 - AMATO MBP; BARBAS CSV; MEDEIROS DM; MAGALDI RB; SCHETTINO GPP; LORENZI-FILHO G; KAIRALLA RA; DEHEINZELIN D; MUNOZ C; OLIVEIRA R; TAKAGAKI TY \& CARVALHO CRR. Effect of a protective ventilation strategy on mortality in the acute respiratory distress syndrome. $\mathbf{N}$ Engl J Med 338: 347-354, 1998.
3 - II CONSENSO BRASILEIRO DE VENTILAÇÃO MECÂNICA. J Pneumol 26, 2000. Supl. 2.

4 - PIERCE LNB. Guide to ventilation and intensive respiratory care. WB Saunders, Philadelphia, 1995, 380 p.

5 - MAC INTYRE NR. New modes of mechanical ventilation. Clin Chest Med 17:411-423, 1996.

6 - PEREL A \& STOCK MC. Handbook of mechanical ventilatory support. Williams \& Wilkins, Baltimore, 1992, $308 \mathrm{p}$.

7 - REVIRIEGO BC \& CRUZ PA. Ventilatión mecánica. Rev Clin Esp 197:13-30, 1997.

Recebido para publicação em 06/07/2000

Aprovado para publicação em 04/05/2001 